\title{
Slot-Filler and Taxonomic Organization: The Role of Contextual Experience and Maternal Education
}

\author{
Li Sheng ${ }^{1} \&$ Boji P. W. Lam ${ }^{1}$ \\ ${ }^{1}$ Department of Communication Sciences and Disorders, University of Texas-Austin, Austin, TX, USA. \\ Correspondence: Li Sheng, Department of Communication Sciences and Disorders, University of Texas-Austin, \\ 2504-A Whitis Ave., A1100, Austin, TX 78712, USA. Tel: 1-512-471-4060. E-mail: 1i.sheng@utexas.edu
}

Received: February 6, 2015

Accepted: March 30, 2015

Online Published: April 24, 2015

doi:10.5539/jedp.v5n1p128

URL: http://dx.doi.org/10.5539/jedp.v5n1p128

\begin{abstract}
Previous studies on children's semantic development suggest a shift from slot-filler to taxonomic organization at around eight years of age. However, these studies typically did not include children of early elementary-school ages (six- or seven-year-old); hence the possibility remains that the shift could have emerged earlier in development. The goal of the present study was to examine the age at which the taxonomic advantage in semantic organization occurs in a cross-sectional sample of children covering a wider age range and elucidate the factors related to the use of different semantic organizational strategies. Forty-six Mandarin-English bilinguals belonging to three age groups (five-, six-, and seven-year-old) were administered category generation task in both slot-filler (e.g., "Name all the zoo animals you can think of.") and taxonomic conditions (e.g., "Name all the animals you can think of."). The taxonomic advantage emerged as early as six years of age. Knowledge of specific slot-filler categories (farm vs. zoo animals) showed different age-related changes. Age and maternal education more consistently predicted performance in taxonomic than slot-filler condition. The slot-filler to taxonomic shift in semantic organization is exhibited across populations of distinct language background in early school-age years. Experience with specific categories and parental input both play important roles in the development of semantic organization.
\end{abstract}

Keywords: maternal education, semantic category, slot-filler, taxonomic organization

\section{Introduction}

A crucial question in cognitive development is how children come to understand general taxonomic categories (Inhelder \& Piaget, 1964; Rosch, 1975). Taxonomic category refers to grouping of objects not limited to specific event schemas but according to functions that cut across various events (Nelson \& Nelson, 1990; Peña, Bedore, \& Zlatic-Giunta, 2002). Taxonomic category requires understanding of features and functions of objects that is not contextually restricted. For example, the animal taxonomy is comprised of entities that differ drastically in both perceptual/biological features (i.e., mammals, reptiles, and insects) and spatial/temporal features (i.e., farm, zoo, and sea animals).

A prominent theory in taxonomic category development posits that children form conventional superordinate taxonomies by combining slot-filler categories (Lucariello \& Nelson, 1985; Nelson, 1988; Nelson \& Nelson, 1990; but see Berger \& Aguerra, 2010 for a different view). Slot-filler category refers to grouping of concepts according to world experiences and specific scripts. Items that are grouped within the same slot-filler are usually objects that share common functions bounded by a specific event. For instance, cereal, eggs, and milk are foods that one often has for breakfast. Objects within the same slot-filler category are thought to have closer spatial/temporal organization (Hudson \& Nelson, 1982). As a result, this event-like organization would be more salient in nature and may help children to process and represent external reality. Once children have acquired several slot-filler categories, such as animals that could be found at the zoo, at home, or on a farm, these initial conceptual structures would interact and help children come to understand the more abstract taxonomic categories that are at a higher hierarchical level. At this level, the conventional superordinate category "animals" encompass a wide range of objects that share more general functional features, such as animacy.

Nelson and colleagues conducted a series of studies to capture the transition from schema-, or slot-filler based organization to taxonomic organization (Lucariello \& Nelson, 1985; Kyratzis, Lucariello, Nelson, \& Greenstein, 
1987; Nelson, 1988). Using a number of tasks such as word-list recall, word associations, and forced-choice picture selection, these researchers repeatedly found that slot-filler organization is a more primary component in children's early category knowledge up until 7 years of age. In turn, the formation of these internal event-based conceptual structures, together with semantic structures embedded in adults' conventional usage of language (e.g., reference to abstract superordinate categories), fuel the understanding of abstract taxonomic conceptual structures. For instance, parents may say "Let's go see some animals" in the context of visiting a zoo, a farm, and a circus, thus alerting the child about the more general category structure through the use of the common superordinate.

In a category generation experiment, K. Nelson and Nelson (1990) directly investigated the transition from slot-filler to taxonomic organization as well as the role of experience in this transition. Nelson and colleague recruited three groups of English-speaking monolingual children who were in lower to lower-middle-class urban schools. The first two groups were five-year-old kindergarteners, with or without preschool experience. The third group consisted of second graders who were eight years of age. Children were assigned to one of two conditions. Children in the slot-filler condition were prompted to generate as many items as they could think of under narrow event-based categories (e.g., animals you see at the zoo; food you have for breakfast). Children in the taxonomic condition were prompted to generate items under general superordinate categories (e.g., name all animals you know; name all food you know).

Although the taxonomic condition was broader in scope than the slot-filler condition, the kindergarteners failed to produce a greater number of items in the taxonomic condition. In fact, examination of raw scores revealed that kindergarteners in the slot-filler condition produced a larger number of items than those in the taxonomic condition. Take the animal category for example, the kindergarteners without preschool experience in taxonomic and slot-filler conditions respectively named an average of 5.9 and 6.3 items; the kindergarteners with preschool experience in taxonomic and slot-filler conditions respectively named 8.9 and 11.3 items. The difference in the latter groups approached statistical significance. On the other hand, the pattern reversed in the second-graders, who generated more animals in taxonomic $(M=17.3)$ than slot-filler $(M=14.0)$ condition. This taxonomic advantage was true also for the clothing category (taxonomic $\mathrm{M}=13.0$; slot-filler $\mathrm{M}=10.6$ ) and was especially strong for the category of food (taxonomic $\mathrm{M}=27.6$; slot-filler $\mathrm{M}=12.3$ ). Follow-up analyses suggested that the five-year-olds were less able to call on multiple event contexts when producing responses in the taxonomic condition than the eight-year-olds. In other words, the older children demonstrated a taxonomic advantage over slot-filler organization because they were able to integrate different slot-fillers when responding in the taxonomic condition (K. Nelson \& Nelson, 1990). These findings were later replicated among five- and eight-year-old Korean-speaking monolingual children (Yu \& Nelson, 1993).

With regard to the role of experience in semantic-conceptual development, K. Nelson and Nelson (1990) made several notable observations. First, the fact that the eight-year-olds had more general and better integrated categories than did the five-year-olds prompted the authors to conclude that the older children's broadened experience with a wider variety of contexts, together with their educational experience may have facilitated more advanced semantic organization. Second, the kindergarteners with preschool experience not only produced more items for each category but also showed better organization of the categories than kindergarteners without preschool experience. This result reinforced the conclusion that school activities and adult-child discourse in educational settings may promote the formation of more abstract categories. Third, all three groups of inner city children had limited experience with farm animals, which may explain the large number of inappropriate responses and a lack of group difference for this category. By contrast, these children had much more experience with zoo animals. This was reflected in the results that all groups had fewer inappropriate responses for this category and that the two more advanced groups (i.e., second graders and kindergarteners with preschool experience) generated more responses than the kindergarteners without preschool experience. The authors argued that these findings support the influence of both natural maturational and experiential factors in explaining the development of categorization.

To the extent that linguistic experience shapes children's semantic-conceptual development, we would expect bilingual children to show differences in this domain from their monolingual peers given bilingual children's unique linguistic experience. To the best of our knowledge, only one published study has used the category generation task with young bilinguals to examine category formation and qualitative change in this population (Peña et al., 2002). There were two methodological changes in Peña et al.'s study over K. Nelson and Nelson's original study. First, instead of recruiting 5- and 8-year-olds like K. Nelson and Nelson did, Peña and colleagues recruited two groups of Spanish-English bilingual children who were respectively 4; 5 to 5; 9 (years; months; 
$\mathrm{M}=5 ; 1)$ and $5 ; 10$ to $7 ; 1(\mathrm{M}=6 ; 5)$ of age. Second, instead of assigning children to either slot-filler or taxonomic condition, Peña et al. elicited responses in both conditions in the bilinguals' two languages. Results indicated an age group by condition interaction and no interaction with language. Older children generated greater items in the taxonomic condition than did younger children in either taxonomic or slot-filler conditions. Older children also generated comparable number of slot-filler items as the younger children. In other words, although the authors did not find a statistically significant difference between taxonomic and slot-filler conditions within the older group of children, they found that the number of items generated in slot-filler condition remained relatively stable across age groups, while the older children generated a greater number of items than the younger children in taxonomic condition. This pattern led Peña and colleagues to suggest that bilingual children around age 6; 5 began to demonstrate the taxonomic advantage, midway between the five- and eight-year age groups included in K. Nelson and Nelson (1990) and Yu and Nelson (1992). Peña et al. further suggested that the bilingual children's more diverse linguistic and event-based experiences may require them to use taxonomic strategies to more effectively organize and access semantic information.

The notion that bilingual children may show the taxonomic advantage at an earlier age than monolinguals is intriguing. However, this literature is inconclusive in that Peña and colleagues did not report finding a significant difference between taxonomic and slot-filler conditions within the same age group, the hallmark pattern of this development shift. Furthermore, it should be noted that Nelson and colleagues only sampled two age levels that were three years apart. Had they sampled the five- to eight-year age range continuously, they could have also found an earlier shift.

The current study aims to determine the age at which the taxonomic advantage in semantic organization occurs in young children. We collected category generation responses in both slot-filler and taxonomic conditions in the dominant language of Mandarin-English bilingual children. To better capture the time frame of this developmental shift, we sampled children who were respectively five, six, and seven years of age. In addition, we focused mainly on the category of animal because this category is less culturally and linguistically constrained than clothes and food (Yu \& Nelson, 1993). For instance, in Mandarin there are types of clothing that do not exist in English (e.g., 旗袍, a traditional Chinese dress with a fitted shape and a high collar). Also, food items that are typical for a particular setting in one language may not be so in another language (Peña et al., 2002; Yu \& Nelson, 1993). Indeed, Yu and Nelson (1993) found that Korean-speaking monolinguals generated fewer items for clothes and food categories than English-speaking monolinguals and attributed these findings to cultural difference.

The second aim of this study is to elucidate factors that contribute to the development of slot-filler and taxonomic organization. Specifically, we examined the contribution of both general maturational (indexed by age) and experiential factors (indexed by amount of language use and level of maternal education) to children's performance in slot filler and taxonomic conditions.

\section{Method}

\subsection{Participants}

Fifty-five Mandarin-English bilingual children between four and seven years of age were recruited from the Chinese community in a large city in North America for a larger-scale study about children's vocabulary and grammar development. No participants were reported to have any sensory or neurologic deficits. This sample included two children with specific language impairment who were excluded from analysis. The primary caregivers were interviewed to obtain information about the children's language profiles that include parent-rated language proficiency and daily language exposure. Language exposure was evaluated in terms of both input and output at school and at home on a typical weekday and typical weekend on hourly basis. To ensure that the bilingual children examined in this study had sufficient bilingual exposure children should use Mandarin and English at least $20 \%$ of the time. Also, in order to be more systematic in the grouping of age, with a range of 12 months for each age groups, only children between $4 ; 7$ to 5; 6 (years; months; hereafter referred to as the five-year-old group, $\mathrm{n}=12$, Mean age $=60.4$ months, $\mathrm{SD}=3.7$ months), $5 ; 7$ to $6 ; 6$ (six-year-old group, $\mathrm{n}=18$, Mean age $=73.3$ months, $\mathrm{SD}=4.0$ months), and $6 ; 7$ to $7 ; 6$ (seven-year-old group, $\mathrm{n}=16$, Mean age $=84.0$ months, $\mathrm{SD}=3.8$ months) were included in subsequent analysis. These criteria resulted in the exclusion of nine children from the original sample. Besides the subjective language proficiency ratings provided by the primary caregivers, all participants were administered picture naming tasks designed by our lab in both English and Mandarin for an objective measure of their language proficiency. 
Table 1 shows that percent of English use for the five-year-old group was significantly lower than for the seven-year-old group and that the former age group also had lower parent-rated English proficiency than the latter. The six-year-old group did not differ from either of the other two groups in English use and proficiency rating. Also, the five-year-old group scored lower than the other two age groups in the English naming tasks. No other statistical difference was found across age groups. Despite the aforementioned statistical difference in English use and proficiency, it should be stressed that the critical age group by condition (slot filler vs taxonomic) interaction that indicates the qualitative change in semantic organization focuses on the presence or absence of difference between conditions within the same age group in the same language. Therefore these between group differences in language use and proficiency should not introduce confounds to this critical interaction.

To document the children's language dominance, we conducted an age (five-, six-, and seven-year-old) by language (Mandarin, English) repeated-measures ANOVA on children's picture naming scores. Results showed a main effect of language, $\mathrm{F}(1,43)=46.12, \mathrm{p}<.001, \eta_{\mathrm{p}}{ }^{2}=.52$, and no other effect or interaction. Across all age groups, naming was more accurate in English $(\mathrm{M}=82.30 \%, \mathrm{SD}=9.6 \%)$ than Mandarin $(\mathrm{M}=61.2 \%, \mathrm{SD}=18.2 \%)$. ANOVA on proficiency rating scores revealed no main effects but an interaction between language and age group, $\mathrm{F}(2,86)=5.19, \mathrm{p}=.01, \eta_{\mathrm{p}}{ }^{2}=.19$. Posthoc tests showed significantly higher ratings in English than Mandarin for the seven-year-old group (Mean difference=.56, $\mathrm{p}<.01,95 \% \mathrm{CI}=.19-.94$ ) and no difference between Mandarin and English proficiency rating in the other two groups. The apparent inconsistency between parental subjective rating and performance on an objective naming task for the five- and six-year-old groups may reflect an earlier shift towards English dominance in picture naming than in general oral proficiency (Sheng, 2014).

Table 1. Means (standard deviations) for demographic characteristics

\begin{tabular}{llllll}
\hline & Five-year-old & Six-year-old & Seven-year-old & P value & Pair-wise comparison \\
\hline Sample size & 12 & 18 & 16 & & \\
Male: Female & $5: 7$ & $9: 9$ & $8: 8$ & & \\
Age in months & $60.4(3.7)$ & $73.3(4.0)$ & $84.0(3.8)$ & $<.001$ & five $<$ six $<$ seven \\
Maternal education in years & $18.3(3.2)$ & $20.2(2.9)$ & $18.1(2.5)$ & $=.077$ & \\
English proficiency & $3.9(0.7)$ & $4.4(0.4)$ & $4.6(0.3)$ & $<.01$ & five $<$ seven \\
Mandarin proficiency & $4.3(0.5)$ & $4.2(0.4)$ & $4.1(0.6)$ & $=.551$ & \\
Percent English use & $50.0(14.7)$ & $61.6(11.1)$ & $62.3(12.7)$ & $=.027$ & five $<$ seven \\
English naming $(\%)^{\mathrm{b}}$ & $74.5(10.8)$ & $84.0(8.4)$ & $86.4(6.5)$ & $<.01$ & five $<$ six; five $<$ seven \\
Mandarin naming $(\%)^{\mathrm{b}}$ & $58.7(17.6)$ & $63.0(17.0)$ & $61.1(20.8)$ & $=.822$ & \\
\hline
\end{tabular}

Notes. a. The language proficiency measure was a rating provided by caregivers using a five-point scale: $5=$ very proficient, $1=$ does not know this language

b. The picture naming task adopted in this study was designed by our lab and included 50 items per language

c. Only paired comparisons that reached statistical significance were listed.

\subsection{Tasks and Procedures}

The category generation tasks used to evaluate knowledge of semantic categories among Mandarin-English bilingual children in this study were modeled after the tasks used by K. Nelson and Nelson (1990) and Peña et al. (2002). However, since these tasks were part of a larger study, time constraints did not allow us to administer all categories as did K. Nelson and Nelson (1990) and Peña et al. (2002). A total of six category generation tasks were administered. There were three taxonomic condition tasks (animals, clothes, and food) in English, and one taxonomic condition task in Mandarin (animals). In addition, there were two slot-filler condition tasks in English (farm animals and zoo animals). The slot-filler condition was not administered in Mandarin. 
Data collection began after the study was approved by the ethics board of a major research university. Examiners included the first author, who is a native speaker of Mandarin and highly proficient in English, and trained graduate or undergraduate speech-language pathology students who were either native speakers of each language or highly proficient bilinguals. Testing took place in participants' homes or in a research laboratory. Tests were administered to children on an individual basis on two separate days with the language of the first session counterbalanced across participants. Each session lasts between 60 and 120 minutes. Children were administered a battery of tasks aimed to measure lexical and grammatical development. Children were allowed to take frequent breaks between tasks. Practice for the category generation task was not provided given the time requirements of the entire session. However, all children showed understanding of the task. Children received monetary compensation and a small prize at the end of each session.

In the taxonomic condition tasks examiners prompted children by saying: "Tell me all the animals/clothes/food you can think of," or "Tell me all the animals/clothes/food you know." In the slot-filler condition tasks examiners prompted children by saying: "Tell me all the animals you would find at the zoo," or "Tell me all the animals you would find on a farm." The order of category administered was counterbalanced.

Examiners gave generic feedback such as, "You are doing a good job," or "You are working hard"; no corrective feedback was given. Tasks were not timed. Examiners gave each child as much time as needed to respond. If the child paused for an extended period of time, examiners used minimal prompts such as "And ...?" or "What else ...?" to elicit additional responses from the child. Once the child indicated they could no longer provide any new items, the examiner continued to the next task.

\subsection{Scoring and Analysis}

The number of correctly generated items was tallied for each individual task. To score the English tasks, each answer was evaluated against a list of acceptable answers developed by Peña et al. as part of the normed Bilingual English Spanish Assessment (BESA) protocol (Peña, Gutierrez-Clellen, Iglesias, Goldstein, \& Bedore, 2014). Repetitions, code-switched responses, as well as any answers that were not on the BESA scoring manual were not counted. For the English tasks, no code-switched responses were observed in any of the bilingual children. The Mandarin task was scored by following the English coding criteria as closely as possible. Two proficient bilinguals coded the responses independently; inconsistencies between coders were resolved through adjudication by the first author.

\section{Results}

\subsection{Comparisons across Languages}

As described earlier, picture naming performance indicated that as a group, these bilingual children were English-dominant. Thus, in the first analysis we compared children's performance in the taxonomic animal task in both English and Mandarin to see if children would again demonstrate English dominance in the current experimental task. We ran a repeated-measure ANOVA with language (English, Mandarin) as within-subject factor and age group as between-subject factor. Total number of correct responses in the taxonomic animal condition in each language was entered as dependent variable. There was a main effect of language, $\mathrm{F}(1$, $43)=5.8, \mathrm{p}=.02, \eta_{\mathrm{p}}{ }^{2}=0.12$, and a main effect of age group, $\mathrm{F}(2,43)=8.2, \mathrm{p}=.001, \eta_{\mathrm{p}}{ }^{2}=.28$, but no language by age group interaction. The language effect indicated that bilingual children in this study generated more responses in English than in Mandarin across all age groups. The age effect was due to a significant difference between the five- and six-year-old groups (Mean difference $=-4.4, \mathrm{p}<.01,95 \% \mathrm{CI}=-1.4$ to -7.4 ) and a difference between the five- and seven-year-old groups (Mean difference $=-4.3, \mathrm{p}<.01,95 \% \mathrm{CI}=-1.3$ to -7.4 ). There was no difference in performance between the six- and seven-year-old groups. We then examined if the performance gap between English and Mandarin category generation was related to the degree of balance in language usage. Task performance gap was calculated by dividing the total number of correct responses in English by the total number of correct responses in Mandarin. Degree of balance in language usage was operationalized as the ratio of English use to Mandarin use. Ratio was calculated by dividing the amount of English use by the amount of Mandarin use. A score of one would indicate balanced category generation performance/balanced use of the two languages; while a score larger than one would indicate better performance in/great use of English. As expected, the correlation between the English/Mandarin category generation performance score and usage ratio was statistical significant, $\mathrm{r}=.35, \mathrm{p}=.02$, indicating that children who had greater use of English also generated greater number of English responses. 
Table 2. Mean number of items (standard derivation) generated for each category generation task

\begin{tabular}{llll}
\hline & Five-year-old & Six-year-old & Seven-year-old \\
\hline Mandarin animal & $4.5(2.6)$ & $8.5(5.7)$ & $8.3(3.2)$ \\
English animal & $5.5(4.3)$ & $10.4(3.3)$ & $10.4(3.7)$ \\
English farm animal & $5.1(2.1)$ & $5.5(1.9)$ & $6.1(1.3)$ \\
English zoo animal & $4.1(2.4)$ & $5.5(3.0)$ & $8.7(2.7)$ \\
English clothes & $3.3(2.7)$ & $6.6(2.7)$ & $6.1(1.7)$ \\
English food & $6.1(4.2)$ & $7.2(2.6)$ & $9.9(5.5)$ \\
\hline
\end{tabular}

\subsection{Comparisons across Conditions}

Given that English is the dominant language in these bilingual children, the following analyses examined the emergence of the taxonomic shift in the children's dominant language. A repeated-measures ANOVA was ran with category condition (taxonomic animal, slot-filler farm animal, and slot-filler zoo animal) as within-subject factor and age (five-, six-, and seven-year-old) as between-subject factor. Number of correct English responses was entered as dependent variable. There was a main effect of age, $F(2,43)=9.56, \mathrm{p}<.001, \eta_{\mathrm{p}}{ }^{2}=.31$, a main effect of condition, $\mathrm{F}(2,86)=24.04, \mathrm{p}<.001, \eta_{\mathrm{p}}{ }^{2}=.36$, and an age group by condition interaction, $\mathrm{F}(4,86)=5.74, \mathrm{p}<.001$, $\eta_{\mathrm{p}}{ }^{2}=.21$. Post-hoc analyses showed that the five-year-olds provided a comparable number of responses in taxonomic and both slot-filler farm and zoo conditions. On the other hand, the six-year-olds displayed better performance in the taxonomic condition than the two slot-filler conditions $(+4.9 \%, \mathrm{p}<.001,95 \% \mathrm{CI}=+2.8 \%$ to $+7.0 \%$ for both taxonomic vs. slot-filler "farm animal" and taxonomic vs. slot-filler "zoo animal" comparisons) and no statistical difference between farm and zoo conditions. The seven-year-olds provided the greatest number of responses in the taxonomic condition, significantly greater than the number in the slot-filler farm animal condition $(+4.4 \%, \mathrm{p}<.001,95 \% \mathrm{CI}=+2.1 \%$ to $+6.6 \%)$. This age group also provided statistically greater number of items in zoo animal than farm animal conditions $(+2.6 \%, \mathrm{p}=.001,95 \% \mathrm{CI}=+0.9 \%$ to $+4.3 \%)$. The gap between zoo animal and taxonomic animal condition was not significant in this age group $(\mathrm{p}=.17)$.

Further analyses of the age group by condition interaction revealed that in the taxonomic condition both the sixand seven-year-old groups generated more items than the five-year-old group $(+4.89 \%, \mathrm{p}=.003,95 \% \mathrm{CI}=+1.5 \%$ to $+8.3 \% ;+4.9 \%, \mathrm{p}=.003,95 \% \mathrm{CI}=+1.4 \%$ to $+8.5 \%)$. However, there was no statistical difference between the two older age groups. For the slot-filler "farm animal" condition, there was no statistical difference among the three age groups. Finally, in the slot-filler "zoo animal" condition the seven-year-old group generated greater number of items than the six-year-old group $(+3.2 \%, \mathrm{p}=.005,95 \% \mathrm{CI}=+0.8 \%$ to $+5.5 \%)$ and the five-year-old group $(+4.6 \%, \mathrm{p}<.001,95 \% \mathrm{CI}=+2.0 \%$ to $+7.2 \%)$. There was no statistical difference between the two younger age groups in this condition.

As shown in Figure 1, the shift towards a taxonomic advantage in category generation is evident at six years of age. Also, despite the different age-related patterns for the two slot-filler categories, it should be noted that the age group by condition interaction persisted when the two slot-filler conditions were combined and compared against the taxonomic condition, $\mathrm{F}(2,43)=5.16, \mathrm{p}=0.01, \eta_{\mathrm{p}}{ }^{2}=.19$. Post-hoc analyses confirmed the patterns shown in the previous analysis that only the two older age groups generated statistically greater number of items in taxonomic than slot-filler conditions. 


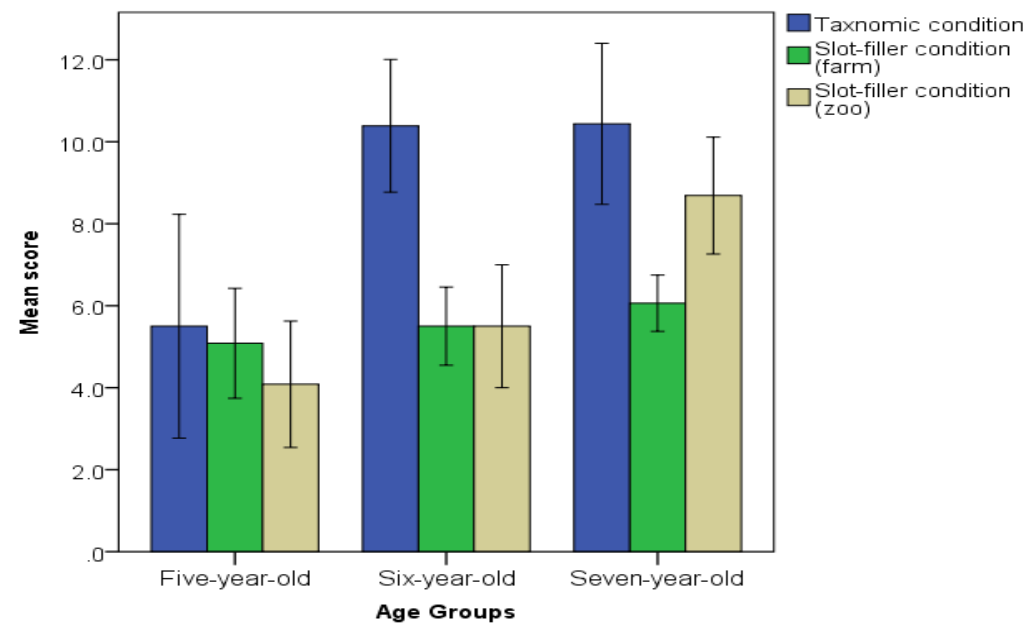

Figure 1. Performance in taxonomic and slot-filler conditions (animals) across age groups

Note. Error bars denote $95 \%$ confidence intervals.

\subsection{Comparisons across Categories}

We then examined whether performance on English category generation tasks in these children would differ across different categories such as food, animals, and clothes. A repeated-measures ANOVA was ran with category (clothes, food, animals) as within-subject factor and age (five-, six-, and seven-year-old) as between-subject factor. Again, there was a main effect of age, $F(2,43)=7.78, p<.001, \eta_{p}{ }^{2}=.27$, with the five-year-old group generating fewer items than both the six- $(-3.1 \%, \mathrm{p}=.009,95 \% \mathrm{CI}=-0.6 \%$ to $-5.6 \%)$ and seven-year-old $(-3.8 \%, \mathrm{p}=.001,95 \% \mathrm{CI}=-1.3 \%$ to $-6.4 \%)$ groups. There was no statistical difference between the two older age groups. In addition, there was a significant main effect of category, $\mathrm{F}(2,86)=17.77, \mathrm{p}<.001$, $\eta_{\mathrm{p}}{ }^{2}=.29$, and a non-significant age group by category interaction $(\mathrm{p}=.058)$. Post-hoc analysis of the category effect showed that the clothes category elicited fewer items than animals $(-3.5 \%, \mathrm{p}<.001,95 \% \mathrm{CI}=-2.2 \%$ to $4.8 \%)$ and food $(-2.4 \%, \mathrm{p}=.002,95 \% \mathrm{CI}=-0.8 \%$ to $-4.1 \%)$. In contrast with K. Nelson and Nelson (1990) who found that children generated the greatest number of items for food and Peña and colleagues (2002) who found that children generated more items in the animal category than the food category in the taxonomic condition; in this study we found no statistical difference between animals and food category. These patterns are displayed in Figure 2 .

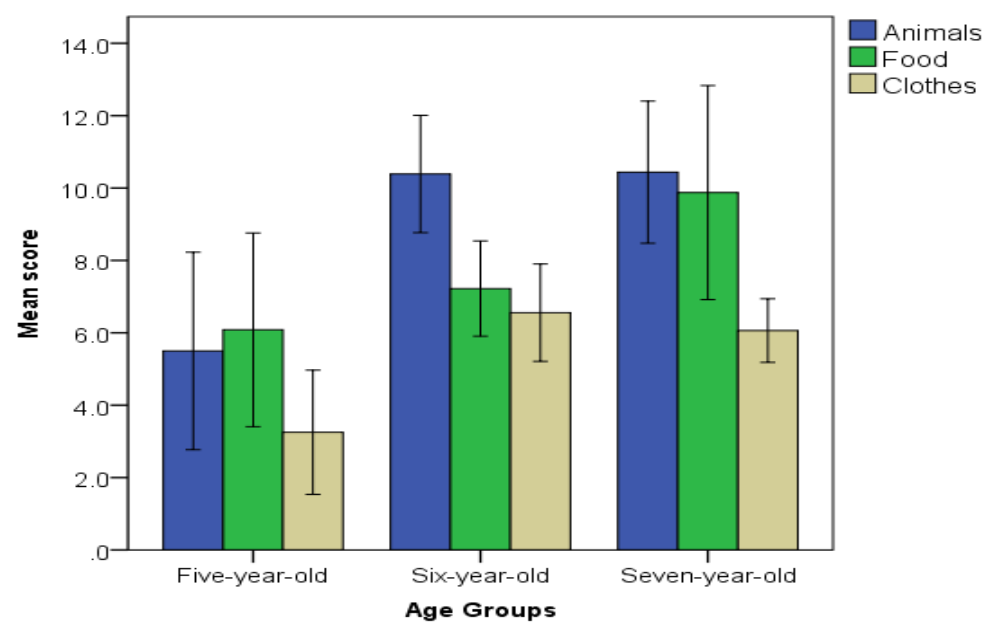

Figure 2. Performance in three different taxonomic categories across age groups

Note. Error bars denote $95 \%$ confidence intervals. 


\subsection{Predictors of Performance}

In the last set of analyses, we examined what factors contributed to children's category generation performance. We ran separate simultaneous regressions on all six category generation tasks, namely taxonomic animals in both English and Mandarin, taxonomic food and clothes in English only, and slot-filler zoo animals and farm animals in English only. Total number of correct responses in each task was entered into the models as outcome variables. In all models, age, maternal education, and amount of usage in English, or Mandarin when applicable, were all mean-centered and entered as explanatory variables. Model refinement was carried out by dropping statistically non-significant variables (using an alpha level of 0.05 ).

Tables 3 and 4 display the results of all six final models, showing only explanatory variables that were statistically significant. There were two patterns. First, age was a robust predictor and accounted for performance in all four taxonomic tasks and the zoo animal slot-filler task. Second, maternal education was statistically significant in three out of four taxonomic tasks, predicting performance in generating animals in both languages and clothes in English. On the other hand, maternal education did not predict performance in either slot-filler task.

Table 3. Summary of simple regression analyses for variables predicting performance in taxonomic conditions $(\mathrm{N}=46)$

\begin{tabular}{|c|c|c|c|c|c|c|c|c|c|c|c|c|}
\hline \multirow[t]{2}{*}{ Variable } & \multicolumn{3}{|c|}{ Food (English) } & \multicolumn{3}{|c|}{ Clothes (English) } & \multicolumn{3}{|c|}{ Animals (English) } & \multicolumn{3}{|c|}{ Animals (Mandarin) } \\
\hline & B & SE B & $\beta$ & B & SE B & $\beta$ & B & SE B & $\beta$ & B & SE B & $\beta$ \\
\hline Age & .13 & .06 & $.29 * *$ & .11 & .04 & $.41^{* *}$ & .22 & .05 & $.52 * *$ & .24 & .06 & $.52^{* *}$ \\
\hline $\begin{array}{l}\text { Maternal } \\
\text { education }\end{array}$ & N.S. & & & .27 & .12 & $.30^{*}$ & .40 & .18 & $.28 *$ & .50 & .20 & $.33^{*}$ \\
\hline $\begin{array}{l}\text { Amount } \\
\text { of usage }\end{array}$ & & N.S. & & & N.S. & & & N.S. & & 14.18 & 4.7 & ${ }_{*}^{.42 *}$ \\
\hline $\mathrm{R}^{2}$ & $8.6 \%$ & & & 24.5 & & & 34. & & & $32.4 \%$ & & \\
\hline $\mathrm{F}$ & $4.13^{*}$ & & & 6.96 & & & 11.0 & & & $6.71^{* *}$ & & \\
\hline
\end{tabular}

Note. ${ }^{*} \mathrm{p}<.05 . * * \mathrm{p}<.01$.

Table 4. Summary of simple regression analyses for variables predicting performance in slot-filler conditions $(\mathrm{N}=46)$

\begin{tabular}{|c|c|c|c|c|c|c|}
\hline Variable & \multicolumn{3}{|c|}{ Zoo Animal (English) } & \multicolumn{3}{|c|}{ Farm Animal (English) } \\
\hline & $\mathrm{B}$ & SE B & $\beta$ & B & SE B & $\beta$ \\
\hline Age & .18 & .04 & $.54 * *$ & & N.S. & \\
\hline Maternal education & N.S. & & & & N.S. & \\
\hline Amount of language use & & N.S. & & & N.S. & \\
\hline $\mathrm{R}^{2}$ & $29.0 \%$ & & & & & \\
\hline $\mathrm{F}$ & $17.99 *$ & & & & & \\
\hline
\end{tabular}

\section{Discussion}

This study examined English category generation performance in Mandarin-English bilingual children. Using a fine-grained age division, we were able to determine the age at which qualitative changes in semantic organization take place in the dominant language of a group of Mandarin-English bilingual children. The results showed that the taxonomic advantage emerged as early as six years of age. We found that age predicted performance in five out of six tasks and maternal education predicted performance in three out of four taxonomic but neither slot-filler tasks. The specificity of the relationship between maternal education and taxonomic task 
performance suggests that mothers with higher education may be providing language input that directly supports the development of taxonomic categories.

The category generation task has elicited consistent findings across studies (K. Nelson \& Nelson, 1990; Peña et al., 2002; Yu \& Nelson, 1993), including ours. Despite the fact that these four studies examined children of distinct language background, including English- and Korean-speaking monolingual children and bilingual children who spoke different first languages (i.e., Spanish and Mandarin), the recurring finding was that children who are five years of age produce comparable numbers of items in taxonomic and slot-filler conditions in this free recall task. This is true even though the former condition is broader in nature thus allowing a greater number of potential answers. The five-year-olds in the current study generated an average of 4.1 and 5.1 items for zoo and farm animal categories; but they appeared unable to integrate these slot-filler categories when prompted by the taxonomic cue, generating only 5.5 items for this condition. The salience of slot-filler category at around the same age found across different populations highlights the important role of event schema in children's spontaneous organization of semantic knowledge. Children across these diverse languages and cultures pay attention to objects that participate in meaningful events in their environments. In turn, knowledge of the role of these objects in familiar events provides a sound basis for object categorization (K. Nelson \& Nelson, 1990).

In contrast to the five-year-olds, the six-year-olds in the current study presented a very different profile. These children nearly doubled their answers from slot-filler to taxonomic condition. This taxonomic advantage over the slot-filler condition was maintained in the seven-year-old group. Recall that Peña and colleagues (2002) had suggested that the Spanish-English bilingual children in their study may begin to demonstrate the taxonomic bias at the mean age of $6 ; 5$, based on the findings of a significant difference in the taxonomic condition between the older (mean age $=6 ; 5$ ) and younger (mean age $=5 ; 1$ ) age groups but a lack of difference in the slot-filler condition. However, the critical difference between the taxonomic and slot-filler conditions within the same age group was not reported. The current study was therefore the first to document the taxonomic advantage in children as young as six. Although it is tempting to suggest that bilingual children may demonstrate an earlier taxonomic advantage than their monolingual peers, it should be noted that previous studies with monolinguals (K. Nelson \& Nelson, 1990; Yu \& Nelson, 1993) did not included six- and seven-year-old children. Had they done so, an earlier shift may have been discovered. A more precise time frame of the taxonomic shift in monolinguals warrants further investigation.

Given the extant evidence that the taxonomic advantage over slot-filler organization is firmly in place by eight years of age and can emerge as early as age six, we now turn to factors that contribute to this developmental change. Existing studies have documented the important role of experience in the development of semantic organization. K. Nelson and Nelson (1990) found that kindergarteners with preschool experience consistently provided a greater number and better organized answers in category generation tasks than those without, suggesting the facilitative role of schooling. K. Nelson and Nelson (1990) also found that kindergarteners and second graders did not differ in the number of farm animals generated, but both second graders and kindergarteners with preschool experience generated more zoo animals than kindergarteners without preschool experience. These findings reflect the influence of exposure to specific event schemas. In a later study, Yu and Nelson (1993) found that Korean-speaking children displayed different response patterns for food and generated fewer items for clothing than English-speaking children, suggesting the influence of language-specific word frequency and possible cultural factors.

In the current study children of all three age groups generated comparable number of items for the slot-filler "farm animal" category; but there was a significant age difference for the "zoo animal" category in that the oldest group generated greater number of items than the two younger groups. The lack of age-related differences for the farm animal category replicated K. Nelson and Nelson's (1990) results and indicated that children growing up in urban environment have limited experience with this particular event schema. Findings from the current study further add to the literature that maternal education, which predicted performance in taxonomic condition, also influences the development of taxonomic categories.

Development of taxonomic category requires children to cut across several established slot-filler categories that do not share the same scripts. This process requires the ability to extract common attributes shared among objects that are less temporally or spatially organized and thus, less immediately obvious to children. More well-educated mothers may engage children in discourse that highlights the common properties of objects in children's own experientially derived knowledge structures. This kind of input may provide children with insights regarding the abstract superordinate-level relationships among concepts thus move them beyond their 
own initial spontaneous category structure. In other words, parents' elaboration of concepts and more sophisticated use of language may play a direct role in the development of taxonomic category. This idea resonates with the finding of a positive relation between children's vocabulary development and mothers' language and literacy skills (Pan, Rowe, Singer, \& Snow, 2005). It also aligns with the finding that vocabulary development from kindergarten to second grade is more closely related to the sophistication of maternal vocabulary use than the sheer quantity of lexical input (Weizman \& Snow, 2001). What our finding suggests is that quality of parental input may also affect categorization development. What is more interesting from our results is the specificity of the influence of maternal education: It predicted performance in taxonomic tasks but none of the slot-filler tasks. This difference further highlights the distinction between slot-filler and taxonomic category. Because the former is based on familiar event schema, it is possible that exposure to daily routines, such as milk and cereal for breakfast, may have already made the shared features of the objects salient enough for young children to spontaneously group them within the same category, therefore lessening the importance of elaboration from parents.

It should be noted that the current study could not specify which aspects of parental input as impacted by maternal education directly influence the development of taxonomic categories. Also, it is possible that the potential influence of maternal conceptual elaboration is not equal across taxonomic categories. As Peña and colleagues (2002) have suggested, the food "scripts" may be more salient than animals and clothing for children due to their everyday experience. This increased salience may facilitate the acquisition of food items within different slot-filler categories and the subsequent development of the food taxonomy. Support for this argument can also be found in K. Nelson and Nelson (1990) wherein children consistently generated more food items than clothing and animal items in both slot-filler and taxonomic conditions. Although our study did not find statistical differences between animal and food as did K. Nelson and Nelson, the food category remains one of the most productive categories across the four studies using the category generation task (K. Nelson \& Nelson, 1990; Peña et al., 2002; Yu \& Nelson, 1993). If the category of food is indeed conceptually less complex and easier to acquire, it would explain why maternal education predicted children's performance for the taxonomy of clothing and animal but not food in our study. Nevertheless, how the quantity and quality of parental language input relates to the development of taxonomic categories of varying levels of conceptual complexity is still an open question that warrants a more fine-grained analysis in the future.

Several specific limitations of the current study bear mentioning. First, we were able to test the children's Mandarin category knowledge with only one task. This limited our ability to observe the bilingual's combined semantic conceptual system. However, for the purpose of determining when the taxonomic advantage may occur in development, the current focus on the bilinguals' dominant language seems appropriate. Second, we tested slot-filler categories for animals only. This limited our ability to generalize the current finding to other categories. Whether or not the slot-filler to taxonomic shift in semantic organization emerges around age six for other natural categories is a question that remains to be answered. Third, the fact that we didn't include monolingual control groups limited our ability to examine the bilingual effect on the development of the taxonomic shift. However, provided that our primary aim was to determine the age at which this shift in semantic organization can occur in young bilingual children, our findings aligned with those in Peña et al. (2002), who studied a population who speak a distinct language pair (i.e., English and Spanish). Finally, future studies are also advised to adopt a double blind design to minimize the possible effect of the high level interaction between experimenters and children during data collection.

\section{Conclusion}

Using a fine-grained age division, our study showed that the taxonomic shift in semantic organization can emerge as early as age six. Performance profiles on the category generation tasks varied across categories, implicating the role of contextual experience. Finally, the influence of maternal education on children's taxonomic performance suggested that sophisticated and instructive language input may help children build their emergent category structure.

\section{References}

Berger, C., \& Aguerra, E. (2010). Dynamic categorization and slot-filler representation in 4- and 6-year-old children. International Journal of Psychology, 45(2), 81-89. http://dx.doi.org/10.1080/ 00207590903281112

Hudson, J., \& Nelson, K. (1983). Effects of script structure on children's story recall. Developmental Psychology, 19(4), 625-635. http://dx.doi.org/10.1037//0012-1649.19.4.625 
Inhelder, B., \& Piaget, J. (1964). The early growth of logic in the child. New York: Harper \& Row.

Lucariello, J., \& Nelson, K. (1985). Slot-filler categories as memory organizers for young children. Developmental psychology, 21(2), 272-282. http://dx.doi.org/10.1037/0012-1649.21.2.272

Nelson, K. (1986). Where do taxonomic categories come from? Human Development, 31(1), 3-10. http://dx.doi.org/10.1159/000273198

Nelson, K., \& Nelson, A. P. (1990). Category production in response to script and category cues by kindergarten and second-grade children. Journal of Applied Developmental Psychology, 11(4), 431-446. http://dx.doi.org/10.1016/0193-3973(90)90019-G

Pan, B. A., Rowe, M. L., Singer, J. D., \& Snow, C. E. (2005). Maternal correlates of growth in toddler vocabulary production in low-income families. Child Development, 76(4), 763-782. http://dx.doi.org/ 10.1111/j.1467-8624.2005.00876.x

Peña, E. D., Bedore, L. M., \& Zlatic-Giunta, R. (2002). Category-Generation Performance of Bilingual Children: The Influence of Condition, Category, and Language. Journal of Speech, Language, and Hearing Research, 45(5), 938-947. http://dx.doi.org/10.1044/1092-4388(2002/076)

Peña, E. D., Gutierrez-Clellen, V. F., Iglesias, A., Goldstein, B. A., \& Bedore, L. M. (2014). BESA: Bilingual English Spanish Assessment. San Rafael, CA: AR-Clinical Publications.

Rosch, E. (1975). Cognitive representations of semantic categories. Journal of Experimental Psychology: General, 104(3), 192-233. http://dx.doi.org/10.1037/0096-3445.104.3.192

Sheng, L. (2014). Lexical-semantic skills in bilingual children who are becoming English-dominant: A longitudinal study. Bilingualism: Language and Cognition, 17, 556-571. http://dx.doi.org/10.1017/ S1366728913000746

Weizman, Z. O., \& Snow, C. E. (2001). Lexical output as related to children's vocabulary acquisition: Effects of sophisticated exposure and support for meaning. Developmental Psychology, 37(2), 265-279. http://dx.doi.org/10.1037/0012-1649.37.2.265

Yu, Y., \& Nelson, K. (1993). Slot-filler and conventional category organization in young Korean children. International Journal of Behavioral Development, 63(1), 1-14. http://dx.doi.org/10.1177/ 01650254930160010

\section{Copyrights}

Copyright for this article is retained by the author(s), with first publication rights granted to the journal.

This is an open-access article distributed under the terms and conditions of the Creative Commons Attribution license (http://creativecommons.org/licenses/by/3.0/). 